\title{
The Synthesis of Anatase Nanoparticles and the Preparation of Photocatalytically Active Coatings Based on Wet Chemical Methods for Self-Cleaning Applications
}

\author{
Dejan Verhovšek, ${ }^{1}$ Nika Veronovski, ${ }^{1}$ Urška Lavrenčič Štangar, ${ }^{2}$ Marko Kete, ${ }^{2}$ \\ Kristina Žagar, ${ }^{3}$ and Miran $\check{C ̌ e h}^{3}$ \\ ${ }^{1}$ Cinkarna Celje, d.d., Kidriceva 26, 3001 Celje, Slovenia \\ ${ }^{2}$ Laboratory for Environmental Research, University of Nova Gorica, Vipavska 13, 5001 Nova Gorica, Slovenia \\ ${ }^{3}$ Department for Nanostructured Materials, Jozef Stefan Institute, Jamova cesta 39, 1000 Ljubljana, Slovenia \\ Correspondence should be addressed to Dejan Verhovšek, dejan.verhovsek@cinkarna.si
}

Received 27 January 2012; Revised 28 March 2012; Accepted 28 March 2012

Academic Editor: Stéphane Jobic

Copyright () 2012 Dejan Verhovšek et al. This is an open access article distributed under the Creative Commons Attribution License, which permits unrestricted use, distribution, and reproduction in any medium, provided the original work is properly cited.

\begin{abstract}
We report on an improved sol-gel method for the production of highly photocatalytic titanium dioxide $\left(\mathrm{TiO}_{2}\right)$ anatase nanoparticles which can provide appropriate control over the final characteristics of the nanoparticles, such as particle size, crystallinity, crystal structure, morphology, and also the degree of agglomeration. The synthesized anatase nanoparticles were characterized using various techniques, such as X-ray powder diffraction (XRD), scanning electron microscopy (SEM), and transmission electron microscopy (TEM), and were tested in coatings for self-cleaning glass and ceramic surfaces. The coatings were prepared using a soft chemistry route and are completely transparent to visible light and exhibit a high photocatalytic effect, which was determined by contact-angle measurements. Finally, it is worth mentioning that both the sol-gel synthesis method and the coating-preparation method are based on a wet chemical process, thus presenting no risk of handling the TiO ${ }_{2}$ anatase nanoparticles in their potentially hazardous powder form at any stage of our development. Low-price, easy-to-handle, and nontoxic materials were used. Therefore, our work represents an important contribution to the development of $\mathrm{TiO}_{2}$ anatase nanoparticle coatings that provide a high photocatalytic effect and can thus be used for numerous applications.
\end{abstract}

\section{Introduction}

Pigmentary titanium dioxide $\left(\mathrm{TiO}_{2}\right)$ exhibits a high refractive index making it a unique white pigment that is not expected to be replaced in either the long or short term. It is mostly used in the production of paints and coatings of every kind, as well as rubbers, plastics, ceramics, papers, foodstuffs, medicine, and many more [1].

With the advent of nanotechnology, the so-called "nano" form of $\mathrm{TiO}_{2}$ has received a great deal of attention, since nanosized particles have many distinctive and enhanced physical and chemical characteristics. Nanosized $\mathrm{TiO}_{2}$ is known for its many applications, which include dyesensitized solar cells [2], UV absorption for surface protection [3], electrochemical splitting of water [4, 5], and various applications in photocatalysis [6-16]. The photocatalytic effect is a well-known characteristic of the nanosized $\mathrm{TiO}_{2}$, having a large surface area capable of absorbing incident UV radiation and transforming it into electrons $\left(\mathrm{e}^{-}\right)$and positive holes $\left(\mathrm{h}^{+}\right)$, which migrate to the surface, resulting in chemical reactions with adsorbed pollutants and oxygen from the air [17]. The preferred crystal structure of the $\mathrm{TiO}_{2}$ photocatalyst is anatase, which can be prepared with larger surface areas than rutile, although having a slightly larger bandgap $(3.2 \mathrm{eV})$ than the latter.

The sulphate production of pigmentary $\mathrm{TiO}_{2}$ is based on the hydrolysis of a titanyl sulphate solution, which results in the formation of a white precipitate, the so-called metatitanic acid, which is an agglomerate of anatase nanoparticles. In our paper we refer to an agglomerate as a particle in which 
the subunits are connected with each other mainly through physical bonding and can be broken when subjected to stronger attraction forces or changes in the surface charge. An aggregate, on the other hand, is a consolidated particle in which smaller crystallites are connected mainly with crystalline bridges. An aggregate cannot be broken down into individual subunits. In view of this we refer to metatitanic acid as an agglomerate, being made up of smaller subunits, aggregates, which themselves are made up of very small anatase crystallites. The crystallite mean size is about 5$6 \mathrm{~nm}$, the aggregate size ranges from 40 to $100 \mathrm{~nm}$, and the agglomerate size is in the order of $1-2 \mu \mathrm{m}[18,19]$. Since the aggregate size is in the nanoregion, we refer to them as nanoparticles. It is very interesting that the size of the anatase nanoparticles can be easily adjusted, simply by controlling the hydrolysis reactions of titanyl sulphate solution [19]. The aggregates that form the metatitanic acid agglomerate are mainly connected through sulphate bridges. The sulphate bridges are electrostatic bonds which arise due to the negative charge of the sulphate ion [19]. When subjected to watersoluble barium salts, the barium ion, having a greater affinity for the sulphate ion, draws the sulphate ions holding agglomerates together and thus breaks them apart into individual anatase nanoparticles. Since the final suspension is acidic, the nanoparticles within it are well dispersed. The suspension is, therefore, very stable, enabling the separation of the nanoparticles from the barium sulphate precipitate which is a result of the reaction between the barium ions and the sulphate ions [19]. There has been very little or no research on determining the photocatalytic activity of anatase nanoparticles in the as-prepared suspension, nor has much research been done on its possible applications. Since metatitanic acid is an intermediate in the production of pigmentary $\mathrm{TiO}_{2}$, and thus prepared in very large amounts when the sulphate process of production is used, it would be very useful if a procedure could be developed to enable the harvesting of anatase nanoparticles directly from it. In our paper we report on such a procedure, enabling an easy and inexpensive production of a stable, acidic suspension of anatase nanoparticles, having a good photocatalytic activity and self-cleaning applications.

\section{Experimental Details}

2.1. Metatitanic Acid Preparation. The metatitanic acid was prepared using the sulphate process [1] in which ilmenite is dissolved with concentrated sulphuric acid (98\%, Cinkarna Celje Inc.) and then hydrolysed in the presence of anatase seeds. The anatase seeds are prepared separately by hydrolysing a titanyl sulphate solution at $80^{\circ} \mathrm{C}$ and are added into the dissolved ilmenite solution. The amount added was varied through $0.1-1.8 \%$ in order to produce different metatitanic types.

2.2. Preparation of a Stable Anatase Nanoparticle Suspension. A stable anatase nanoparticle suspension was prepared by mixing the metatitanic acid and a barium chloride solution (p.a., Fluka) in the molar ratio Ti: $\mathrm{Ba} \sim 30: 1$. The molar concentration of the barium chloride used in this case was
$0.5 \mathrm{M}$. The concentration of barium chloride was varied between 0.25 and $1.4 \mathrm{M}$ in order to prepare barium sulphate particles of varying sizes. Barium chloride was added to the metatitanic acid dropwise at room temperature while mixing at $200 \mathrm{rpm}$. The reaction mixture was stirred for around 15 minutes. Afterwards, the barium sulphate was removed by means of centrifugation ( $30 \mathrm{~min}$ at $3000 \mathrm{rpm}$ ). After the centrifugation a precipitate of barium sulphate was present on the bottom of the vial, while the $\mathrm{TiO}_{2}$ suspension exhibited no sedimentation, thus allowing a simple separation via decantation.

2.3. Preparation of Anatase Self-Cleaning Coating. In order to prepare a stable colloid used for the final coating application that exhibits good adhesion, mechanical stability, and also superhydrophilicity, a soft chemical method was employed. The anatase was used in the form of an acidic suspension prepared as described above. To the acidic anatase suspension $(5 \mathrm{~mL})$, water $(40 \mathrm{~mL})$, ethanol (Fluka) $(40 \mathrm{~mL})$, and a silica binder solution $(400 \mu \mathrm{L})$ (made from either of the two silica sources, that is, colloidal silica Levasil (200/30\%) and TEOS precursor) were added and stirred until a homogenous mixture formed [20]. The molar ratio of $\mathrm{Ti}$ and $\mathrm{Si}$ in the final suspension is $1.3: 1$. The as-prepared suspension is very stable and remains so for a period of about six months. The as-prepared suspension was used to prepare self-cleaning coatings using various techniques, such as spraying and dip coating onto various substrates. No thermal treatment of the resulting coatings at elevated temperatures was required.

The coatings prepared differ in the size of anatase nanoparticles used. NTi1 and both NTi2 and NTi3 were prepared with anatase nanoparticles synthesized with a seeding volume of 0.3 and $0.6 \%$, respectively.

Coatings NTi4, NTi5, and NTi6 were prepared with anatase nanoparticles synthesized with a seeding volume of $1.8,0.6 \%$, and $0.3 \%$, respectively, and were used to test the effect of particle size on the photocatalytic activity of the final coating. The three coating types were also thermally treated at $450^{\circ} \mathrm{C}(30 \mathrm{~min})$ to achieve a good adhesion on the glass substrate.

2.4. Characterization Methods. For X-ray powder diffraction (XRD) investigations the suspensions were neutralized after the reaction, filtered, washed with distilled water, and dried at $80^{\circ} \mathrm{C}$ in order to acquire $\mathrm{TiO}_{2}$ powder that was used for characterization. The $\mathrm{TiO}_{2}$ powder was pressed into a pellet and the spectra recorded from $10^{\circ}$ to $70^{\circ}$ (2-theta angle) with a $\mathrm{CuK}_{\alpha}$ radiation $(\lambda=1.5418 \AA)$ using a CubiX PRO PW 3800 instrument (PANalytical). The $\mathrm{TiO}_{2}$ crystal structure was identified using the X'Pert Data Viewer software.

Scanning Electron Microscopy (SEM) studies (Jeol JSM7600F, Jeol Ltd., Tokyo, Japan) and Transmission Electron Microscopy (TEM) studies (Jeol JEM-2100, Jeol Ltd., Tokyo, Japan) were used to estimate their morphology and dimensions. The samples for the TEM observations were ultrasonically dispersed and placed onto lacey, carboncoated nickel grids.

The optimum amount of barium chloride to be added was determined by using Dynamic Light Scattering (DLS) 
measurements (Zeta PALS, Brookhaven). The samples for DLS measurement were prepared by dispersing a single $\mathrm{TiO}_{2}$ suspension droplet into $50 \mathrm{~mL}$ of distilled water. The final sample was translucent. The sample was poured into a plastic vessel $(10 \mathrm{~mL})$ which was transferred to the instrument cell where it was analyzed at a fixed angle of $90^{\circ}$ using a laser with a wavelength of $660 \mathrm{~nm}$.

The zeta potential of $\mathrm{TiO}_{2}$ nanoparticles was determined by Zeta PALS (Brookhaven). The samples for zeta potential measurement were prepared by dispersing a single $\mathrm{TiO}_{2}$ suspension droplet into $50 \mathrm{~mL}$ of distilled water and mixing it to form a translucent suspension. The electrophoretic mobility was converted to zeta potential using a PALS Zeta Potential Analyzer software and was based on the Smoluchowski approximation.

Photocatalytic activity measurements were performed using the contact-angle method $[21,22]$. The $\mathrm{TiO}_{2}$ anatase suspension was used to prepare an anatase nanoparticle layer, which was done by dip-coating microscope glass slides $(76 \times$ $26 \mathrm{~mm}$, Menzel-Gläser) into an anatase suspension (100 g/L) (dipping speed of 70 or $100 \mathrm{~mm} / \mathrm{min}$ ). Dip coating of the microscope glass slides was performed only once. The layers were used as-prepared or were additionally thermally treated $\left(450^{\circ} \mathrm{C}, 30 \mathrm{~min}\right)$ in order to achieve better adhesion onto the glass substrate and also to improve the crystallinity of the anatase nanoparticles. Methyl stearate (p.a., Fluka) (0.2 M solution in hexane) was used as a test organic pollutant and was additionally deposited onto the anatase layers using dip coating $(100 \mathrm{~mm} / \mathrm{min})$. After the addition of the methyl stearate the contact angle was measured, while the layers were illuminated with UVA (CLEO light source, $20 \mathrm{~W}, 438 \mathrm{~mm}$ $\times 26 \mathrm{~mm}, \lambda 355 \mathrm{~nm}$, Philips) of ca. $2 \mathrm{~mW} / \mathrm{cm}^{2}$ irradiation intensity. Real-time contact-angle measurements provided a quantitative measurement of the photocatalytic activity of different anatase samples. It was determined for anatase nanoparticles of various sizes.

\section{Results and Discussion}

3.1. Metatitanic Acid Preparation. Metatitanic acid was produced when a titanyl sulphate solution hydrolysed in the presence of a certain amount of anatase seeds. Anatase seeds are crucial for the hydrolysis of the titanyl sulphate solution, since seeding provides better control over the final hydrolysis product. These seeds can have many distinctive benefits $[18,19,23]$ :

(i) they promote the precipitation process;

(ii) they control particle formation and ensure a narrow size distribution of the synthesised nanoparticles;

(iii) they increase the reaction yield;

(iv) they provide an active surface, which can greatly accelerate the crystallization process, since crystallization on a solid-solid interface is much easier than nucleation in a liquid phase;

(v) they decrease the activation energy of a reaction, thus further promoting crystallization.
The inoculation of a reaction mixture with suitable seeds provides all the above-stated benefits through a process of heterogeneous nucleation. Since the seeds used in our reactions are of the same crystal structure as the final product, we call such heterogeneous nucleation a secondary nucleation.

In order to better understand the effect of seeding on the final product, we conducted experiments where we varied the quantity of seeds added to the titanyl sulphate solution. According to the literature, the variation of the seeding volume should directly affect the size of the primary aggregates (nanoparticles) [19]. The SEM results in Figures 1(a)-1(e) indeed showed that larger seeding volumes tend to produce smaller anatase nanoparticles, which are composed of a smaller number of crystallites. On the other hand, smaller seeding volumes produce anatase nanoparticles composed of a larger number of crystallites. It was concluded that one can effectively control the size of the anatase nanoparticles by changing the amount of added anatase seeds. This result also demonstrates that the seeds directly control the nucleation process via secondary nucleation mechanisms.

The theory of heterogeneous nucleation states that the free-energy change needed for the nucleation to occur depends on the wetting angle in a liquid-solid system. In the case of secondary nucleation, the seeds of the same material as the one being crystallized are used; therefore, the affinity in the liquid-solid system is complete. This means that the contact angle becomes zero and that the free energy of nucleation is also zero. In this case no additional nuclei are formed in the supersaturated reaction medium when it is seeded [24]. This is in direct agreement with the observed results of our experiments. Since no additional nuclei were produced after the inoculation with the seeds, they are solely responsible for particle formation and thus also determine their size. As stated previously [19] the seeds added could act as centres for nucleation of the newly formed nanoparticles. It was shown that the number of nanoparticles formed is of the same order of magnitude as the number of crystals inoculated as seeds. This suggests that every nanoparticle arises from a single seed particle, as shown in Figure 2.

The seeds used in the synthesis are large agglomerates of very small anatase crystallites, as shown in Figure 3(a). It is very likely that they break up when added to the titanyl sulphate solution, since their surface charge changes because of the change in the $\mathrm{pH}$ value.

Zeta potential measurement of the anatase seeds was performed by using a diluted water suspension $(\sim 5 \mathrm{~g} / \mathrm{L})$ which was titrated with sodium hydroxide solution $(1 \mathrm{M})$. The titration was done starting at an acidic $\mathrm{pH}$ value $(\sim 2.5)$ and raised to $\sim 10.5$. As seen in the diagram of zeta potential measurements in Figure 3(b), the seeds exhibit the isoelectric point (IEP) at a $\mathrm{pH}$ value of about 5 , which is very near to the actual $\mathrm{pH}$ of the seed suspension that was used. The IEP measured for anatase seeds is lower than expected for anatase which is around 6 [25]. The observed difference could have arisen because of surface impurities such as anions that were adsorbed onto the $\mathrm{TiO}_{2}$ surface. Sulphate anions adsorb onto the $\mathrm{TiO}_{2}$ surface and may reduce the IEP value, as was observed in our measurements [26]. 


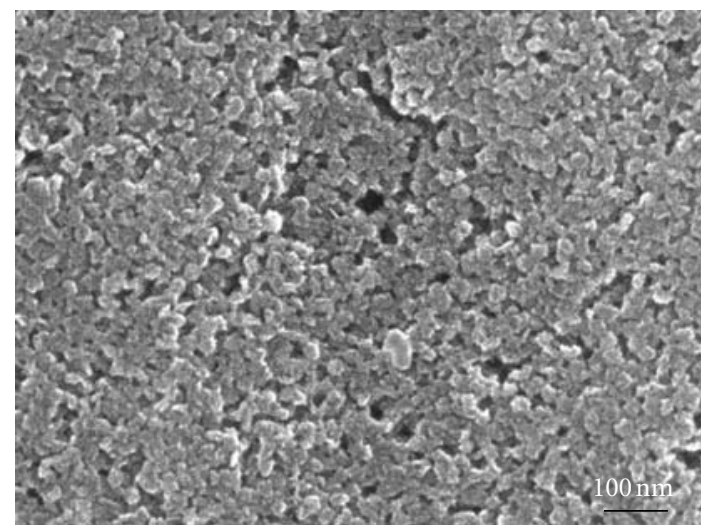

(a)

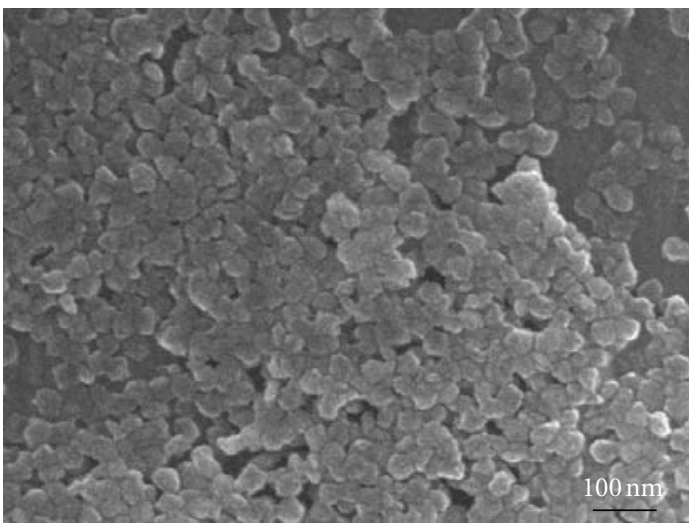

(c)

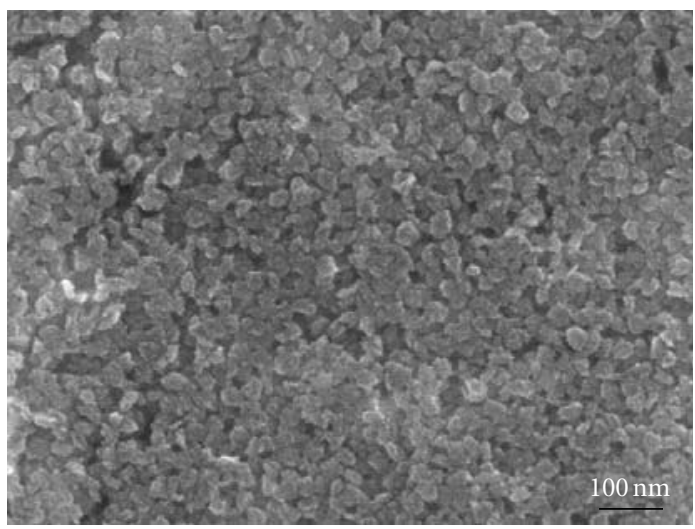

(b)

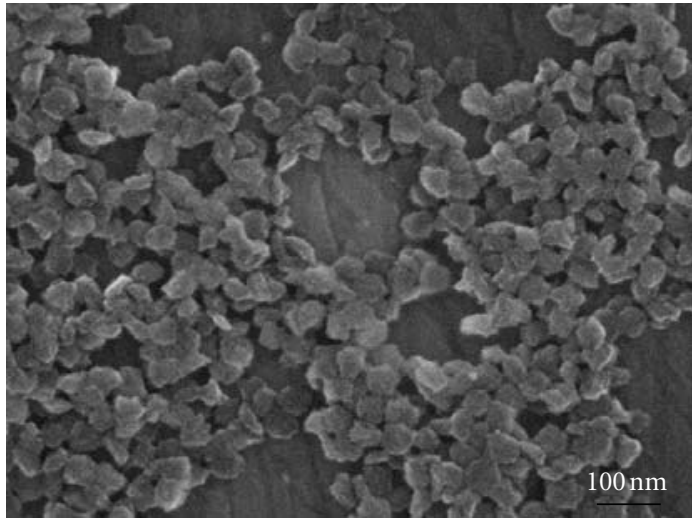

(d)

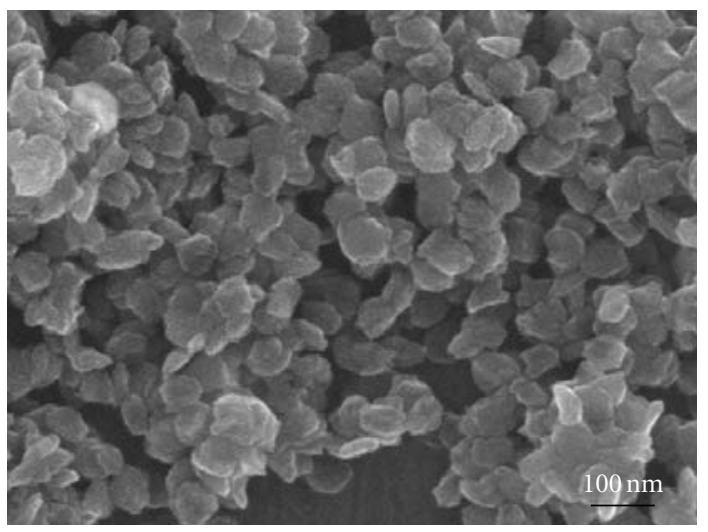

(e)

FIGURE 1: SEM images of anatase nanoparticles produced by the hydrolysis of a titanyl sulphate solution when different seeding volumes were used. The quantity of seeds (regarding the $\mathrm{TiO}_{2}$ content in titanyl sulphate in wt $\%$ ) was (a) $1.8 \%$, (b) $0.9 \%$, (c) $0.6 \%$, (d) $0.3 \%$, and (e) $0.1 \%$.
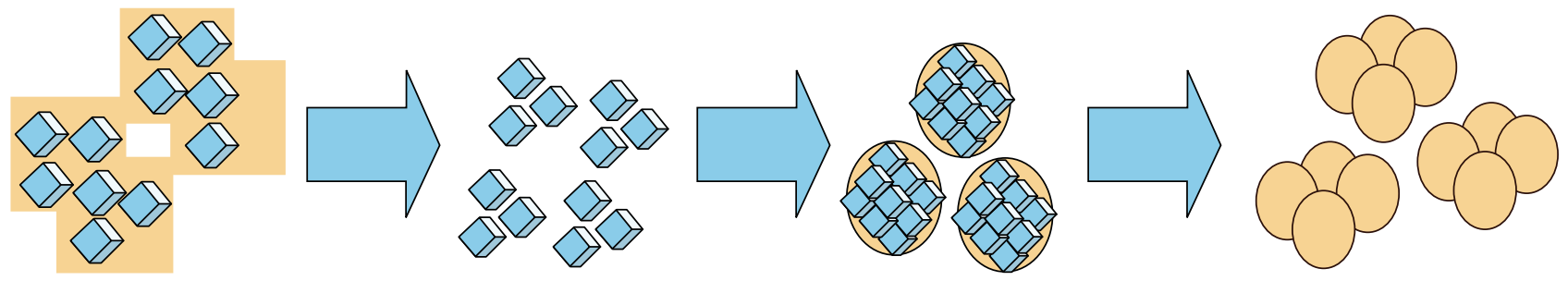

FIGURE 2: Scheme representing a possible mechanism of particle formation via secondary nucleation using seed inoculation [18]. 


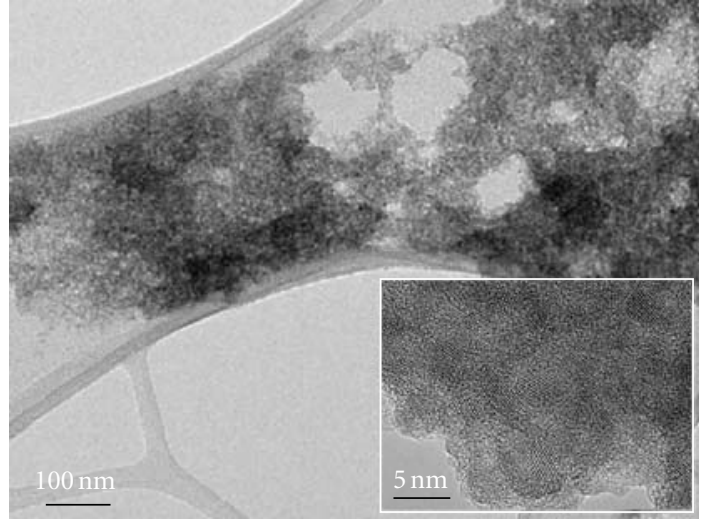

(a)

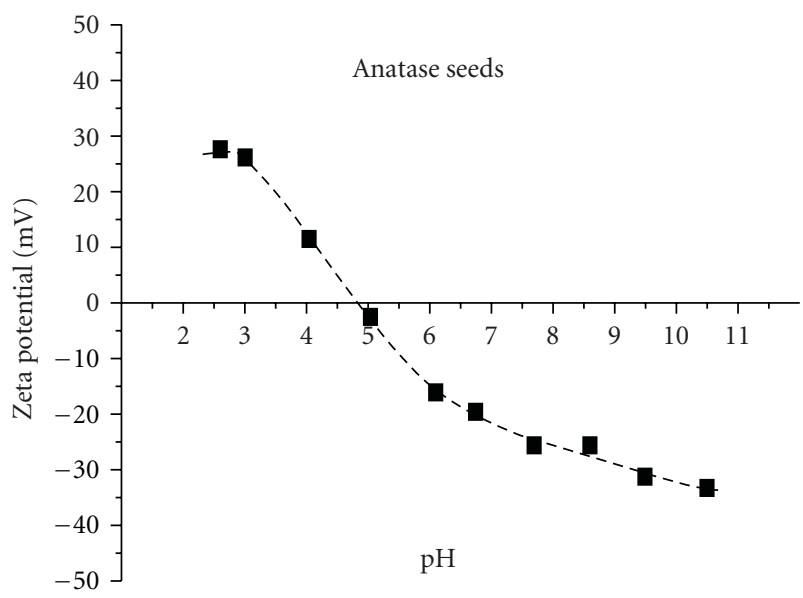

(b)

FIGURE 3: (a) Bright-field TEM image of anatase seed agglomerate. Inset shows high-resolution TEM image of anatase seeds in which smaller, approximately $5 \mathrm{~nm}$ crystallites of anatase are clearly visible. (b) Zeta potential measurement of anatase seeds used to inoculate titanyl sulphate solution. The isoelectric point of the anatase seeds is around the $\mathrm{pH}$ value of 5 , which is also the $\mathrm{pH}$ value of the seed suspension used for inoculation.

Nevertheless, it is reasonable to assume that the particles in the anatase seed suspension are highly agglomerated since its $\mathrm{pH}$ value is near the $\mathrm{pH}$ value of the observed IEP. But when they are added into the acidic titanyl sulphate solution, the $\mathrm{pH}$ drops rapidly (below 1 ). Consequently the zeta potential becomes more positive, which may give rise to deagglomeration of the seed particles into smaller particles, or even individual crystallites.

The mechanism of particle formation during seeding has been researched extensively [27-29]. Qian and Botsaris [29] presented the so-called "embryo coagulation secondary nucleation (ECSN)" model, which combines classical nucleation theory with colloid science. The model states that the supersaturated solution already contains a large number of embryos. When the seeds are inoculated the embryos migrate to the surface of seeds, where they gather in large numbers which then promotes the formation of clusters that are

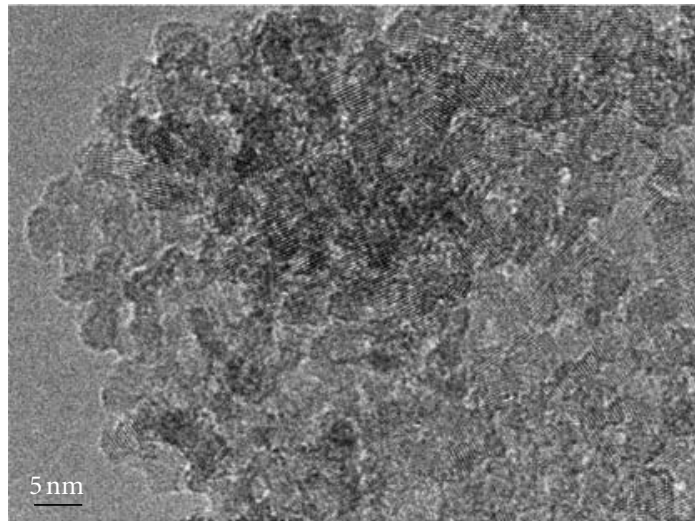

(a)

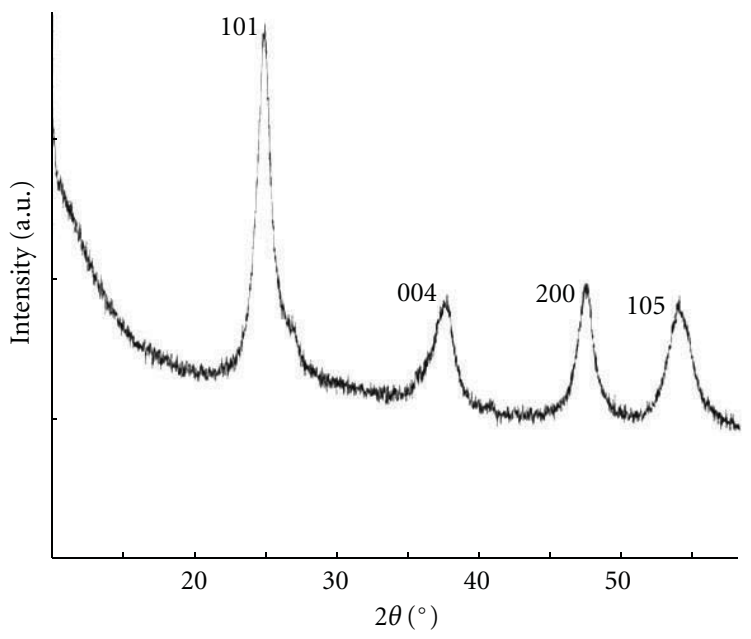

(b)

FIgURE 4: (a) TEM image of an anatase nanoparticle showing its polycrystalline (aggregated) nature. The individual crystallites are approximately $5 \mathrm{~nm}$ in size and they are aggregated into an anatase nanoparticle. (b) X-ray powder diffractogram of the anatase nanoparticles.

larger than the critical nucleus size. Afterwards, the formed nuclei can be swept away from the surface by shear forces which are the result of solution mixing and the consequent collisions between particles. The freed nuclei can then initiate secondary nucleation or they can remain attached to the seed and form a polycrystalline aggregate (nanoparticle). In any case, highly consolidated polycrystalline particles are formed with a narrow size distribution (Figure 1 and Figure 4(a)). A high-resolution TEM image of an individual anatase particle is shown in Figure 4(a), where individual crystallites of approximately $5 \mathrm{~nm}$ in size are clearly visible. From the X-ray diffractogram in Figure 4(b) it is evident that the produced particles have the anatase crystal structure. It also has to be noted that a small amount of rutile $\mathrm{TiO}_{2}$ is present. The shoulder on the right-hand side of the (101) anatase peak corresponds to (110) peak for the rutile $\mathrm{TiO}_{2}$. A small amount of rutile is present in metatitanic acid and is the consequence of production procedure. 
3.2. Formation of a Stable Nanoparticle Suspension. The metatitanic acid that was used to carry out the experiments was made by $0.6 \%$ seed inoculation amount. As was already mentioned in Section 1, the metatitanic acid is made up of the anatase aggregates presented in Figure 4, which are connected into large agglomerates through sulphate ion bridges. In order to produce a stable suspension of anatase nanoparticles, sulphate bridges have to be removed which is possible with the addition of an appropriate amount of barium chloride. Since the barium ion bonds with the sulphate ion that holds the anatase aggregates together, it is very important to add enough of the barium chloride solution in order to remove most of the sulphate ion bridges and thus form a finely dispersed and stable suspension. The optimal addition of barium chloride solution was defined by performing DLS measurements as shown in Figure 5. If the volume added is too low, the agglomerate breakage is not sufficient and the average particle size is around $110 \mathrm{~nm}$ (red curve). By increasing the volume addition of barium chloride solution, the agglomerates break and the average size attained is around $50 \mathrm{~nm}$, which coincides very well with the actual particle size observed by SEM (Figure 1(c)).

From the obtained results it can be concluded that the optimal amount of barium chloride is in the molar ratio $\mathrm{TiO}_{2}: \mathrm{Ba}$ of approximately $30: 1$. This ratio enables the production of very stable anatase nanoparticle suspensions, which can be effectively separated from barium sulphate via various separation procedures, such as centrifugation.

The separation of barium sulphate and $\mathrm{TiO}_{2}$ nanoparticles is quantitative since $\mathrm{TiO}_{2}$ nanoparticles exhibit a high zeta potential at acidic $\mathrm{pH}$ values which prevents their sedimentation. The zeta potential of the $\mathrm{TiO}_{2}$ nanoparticles is shown in Figure 6.

On the other hand, the barium sulphate particles are larger and settle when centrifugation is applied. This was also confirmed with XRD (Figure 7) analysis of the settled material which is pure barium sulphate.

This is important since the method allows the production of a very stable $\mathrm{TiO}_{2}$ acidic suspension without a byproduct being present. The concentration of $\mathrm{TiO}_{2}$ in the final suspension is dependent only on the amount of metatitanic acid that is used. The transformation of the metatitanic acid into $\mathrm{TiO}_{2}$ nanoparticle suspension has a very high yield $(\sim$ $100 \%$ ) since only a sufficient amount of barium chloride has to be added in order to remove the sulphate ions holding the anatase nanoparticles together in the metatitanic acid agglomerate.

It is also interesting to note that by careful addition of a barium chloride solution almost monodispersed particles of barium sulphate are formed (Figure 8). They are in the size range of $0.5 \mu \mathrm{m}$ to $1 \mu \mathrm{m}$, which depends on the concentration of barium chloride used. As expected, the barium sulphate particles form according to the classical theory of nucleation, where control over the particle size can easily be achieved by controlling the solute concentration (supersaturation). If a concentrated barium chloride solution was used (Figure 8(a)), smaller particles formed since supersaturation was larger and thus more particles were

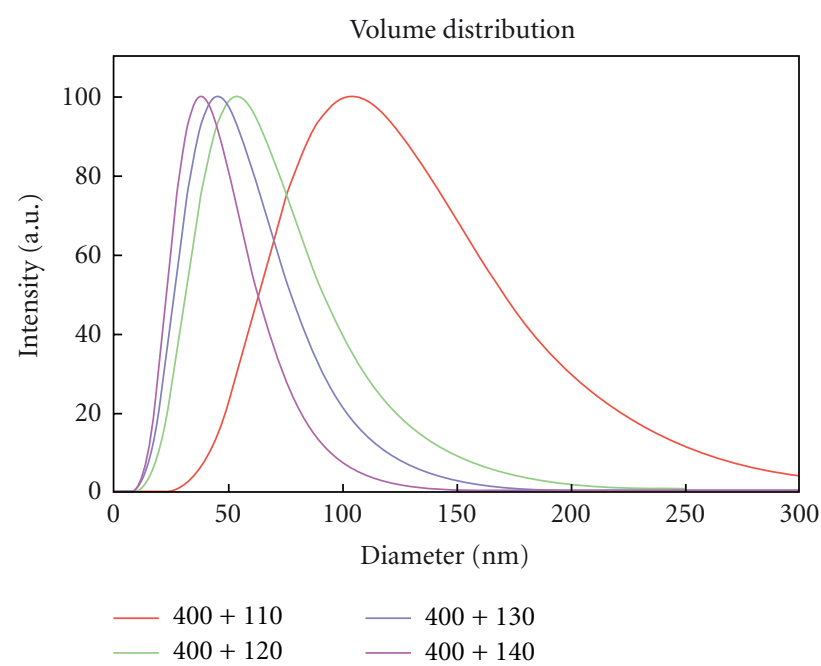

FIGURE 5: DLS measurements of nanoparticle suspensions that were made from metatitanic acid ( $0,6 \%$ seed quantity) and the subsequent addition of a barium chloride solution. The curves correspond to DLS measurements of nanoparticle suspensions prepared using different amounts of barium chloride solution that were added to the metatitanic acid. Namely, $400 \mathrm{~mL}$ of metatitanic acid suspension was mixed with $110 \mathrm{~mL}$ of $0,5 \mathrm{M}$ solution of barium chloride, $120 \mathrm{~mL}, 130 \mathrm{~mL}$, and $140 \mathrm{~mL}$, respectively.

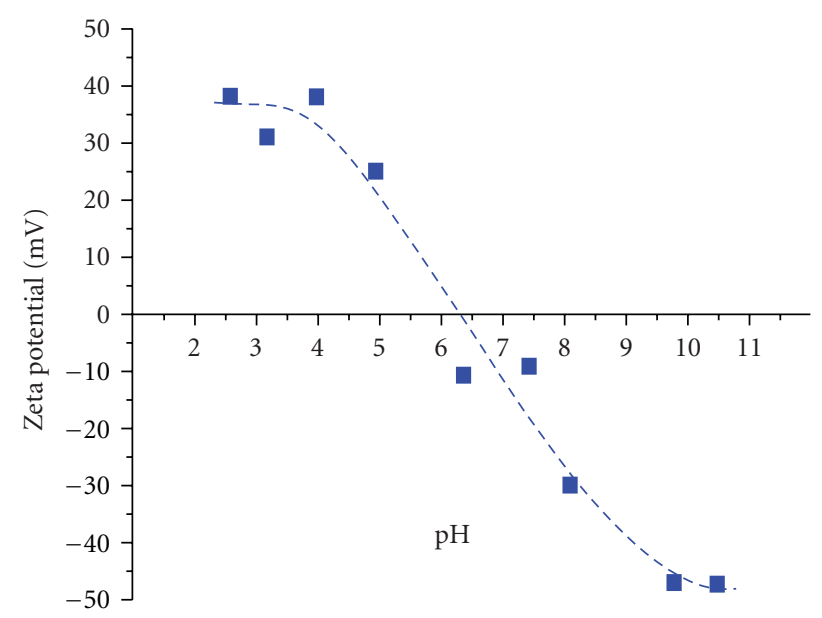

FIGURE 6: The change in zeta potential for anatase nanoparticles when titrating the acidic suspension with sodium hydroxide solution. The zeta potential is large for the acidic suspension but drops when sodium hydroxide is added. The IEP seems to be at the $\mathrm{pH}$ value of about 6 which is in accordance with the results found in literature [25].

formed. On the other hand, if a diluted barium chloride solution was used (Figure 8(b)), then the particles were bigger since supersaturation was lower.

3.3. Characterization of the Photocatalytic Activity of Anatase Nanoparticle Suspensions and Their Application as SelfCleaning Surfaces. The photocatalytic activity of anatase nanoparticles in self-cleaning coatings was determined using 


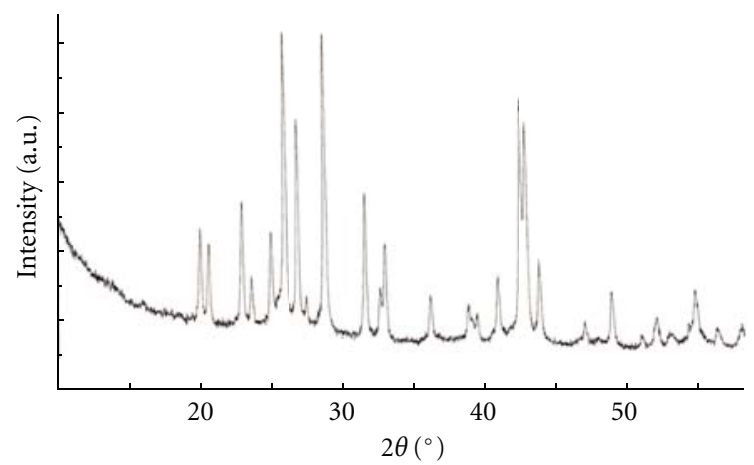

Figure 7: The X-ray powder diffractogram of the material collected after the centrifugation cycle was finished. The diffractogram exhibits all the peaks for barium sulphate while no observable peaks for anatase are evident.

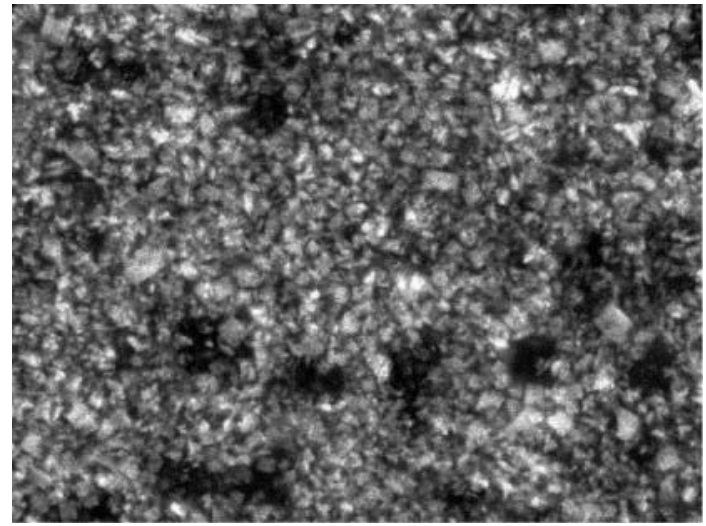

(a)

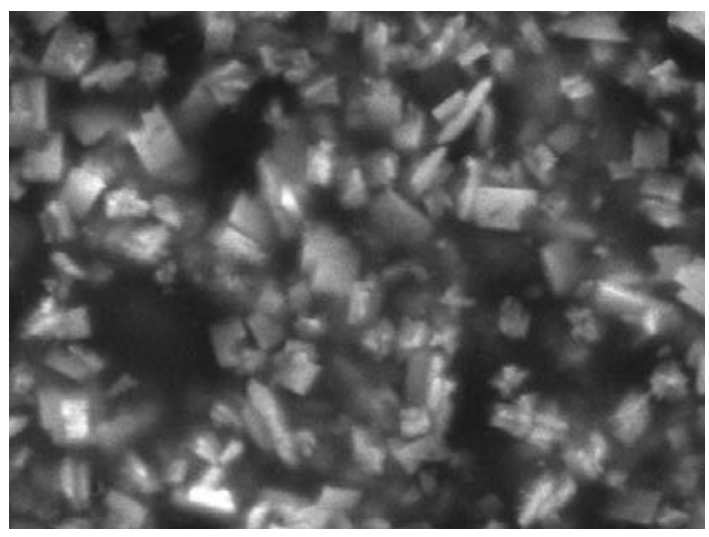

(b)

FIGURE 8: SEM image of barium sulphate particles. (a) Barium sulphate particles formed when a concentrated solution of barium chloride was used. (b) Barium sulphate particles formed when a diluted solution of barium chloride was used.

contact-angle measurements. Figure 7 shows that the samples NTi1 and NTi2 decompose the methyl stearate test pollutant rapidly, since the water contact angle fell in less than one hour of UVA irradiation from initial hydrophobic value, characteristic for the fatty deposit, to the final hydrophilic value, characteristic for clean and hydroxylated
$\mathrm{TiO}_{2}$. The sample coating NTil performed slightly better than the sample coating NTi2, which can be attributed to the fact that the layer thickness differs for the two samples. This could be due to the fact that the anatase nanoparticles differ in size since they were prepared using 0.3 and $0.6 \%$ seeding volume, respectively. However, the difference in layer thickness was not examined. The slight difference in the photocatalytic activity of the NTi1 and NTi2 coatings could also be a direct result of the fact that anatase nanoparticles differ in size. The anatase nanoparticles used for NTi1 coating are depicted in Figure 1(d), the anatase nanoparticles used for NTi2 coating in Figure 1(c). Our results indicate that the anatase nanoparticles depicted in Figure 1(d) could be slightly more photocatalytically active than the anatase nanoparticles depicted in Figure 1(c). However, raw anatase nanoparticles were not analysed for their photocatalytic activity, and therefore the difference in the photocatalytic activity for the NTi1 and NTi2 coating cannot be addressed adequately. Sample coating NTi3 performed worse even though the coatings were additionally thermally treated in order to enhance the particle crystallinity and adhesion to the substrate. Such worsening in performance can be attributed to the fact that a thermal treatment was used. It is possible that during the treatment the nanoparticles present in the coating became larger due to sintering, which lowered their specific surface and consequently the photocatalytic activity of the coating as a whole. Since anatase nanoparticles are well crystallised at the beginning, the thermal treatment does not contribute to their crystallinity and thus does not enhance it (Figure 9).

As one can see (Figure 10) the photocatalytic activity of the anatase nanoparticles depends greatly on the synthesis procedure, that is, on the seeding volume used for the secondary nucleation during the nanoparticle synthesis. Although the nanoparticles synthesized using the $1.8 \%$ seeding volume (NTi4 coating) are smaller in size and thus have a greater specific surface, they performed worse in comparison to the nanoparticles that were synthesized using the $0.6 \%$ seeding volume (NTi5 coating). This can be explained by the fact that the hydrolysis and polycondensation reactions during the synthesis are carried out more rapidly when using a greater seeding volume, which consequently influences the overall crystallinity of the final product. Since the hydrolysis/polycondensation reactions were very fast when the $1.8 \%$ seeding volume was used, it can be assumed that the product crystallinity is worse since the kinetics of the reaction do not allow the crystal structure to be fully developed during the synthesis. Therefore, smaller seeding volumes are necessary and provide a better nanoparticulate anatase which is suitable for self-cleaning applications. However, it seems that a smaller seeding volume of $0.3 \%$ produces a photocatalytically more active anatase than the $0.6 \%$ seeding volume since the NTi6 coating (anatase prepared with $0.3 \%$ seeding volume) performs better than the NTi5 coating (anatase prepared with $0.6 \%$ seeding volume).

Self-cleaning coatings were also tested in real-life environmental conditions (8-month testing period with seasonal climate changes (summer-spring)). Self-cleaning coatings 


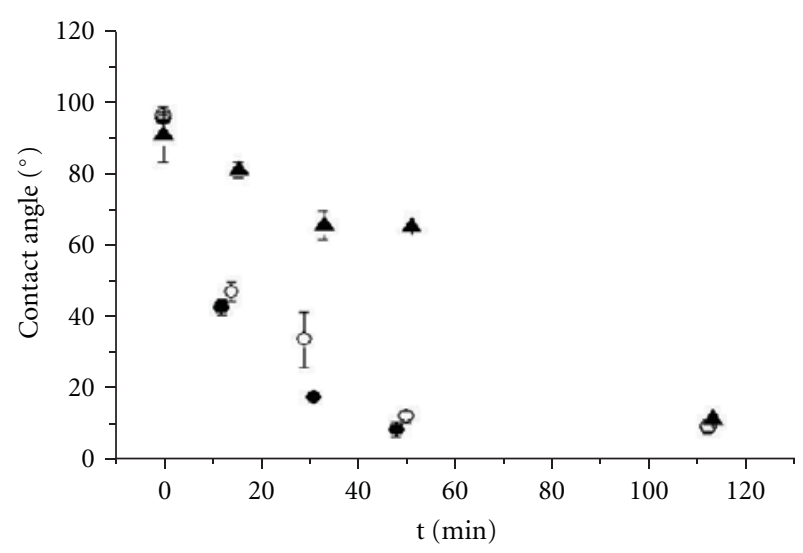

- N-Til (anatase, $10 \mathrm{~cm} / \mathrm{min}$, dried at room temperature)

O N-Ti2 (anatase, $7 \mathrm{~cm} / \mathrm{min}$, dried at room temperature)

- N-Ti3 (anatase, $10 \mathrm{~cm} / \mathrm{min}$, dried at $400^{\circ} \mathrm{C} 30 \mathrm{~min}$ )

Figure 9: Contact-angle measurements for self-cleaning coatings prepared from anatase nanoparticles presented in Figure 1(c) using a soft chemical method. Water contact angles were measured after different times of irradiation of the coatings covered by methyl stearate layer.

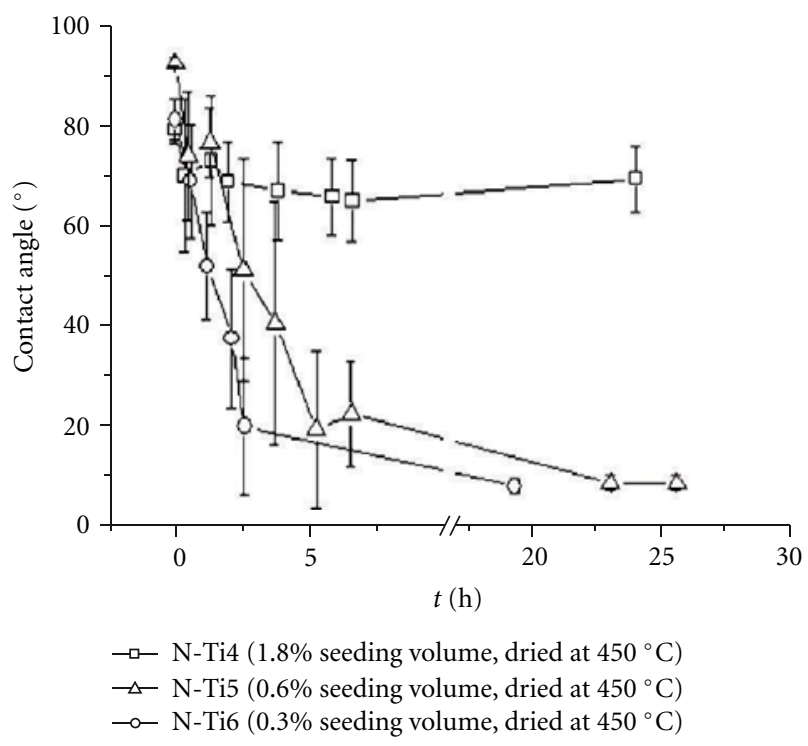

Figure 10: Contact-angle measurements for different coatings prepared from anatase nanoparticles using a soft chemical method. The anatase nanoparticles were prepared using different seeding volumes during the synthesis and therefore differ in size. Water contact angles were measured after different times of irradiation of the coatings covered by methyl stearate layer.

were applied on a glass surface (Figure 11) and exposed to changing weather conditions over a period of 8 months. The coatings remained photocatalytically active and mechanically stable over the whole testing period.

This was tested by performing swift quantitative tests for photocatalytic performance using the resazurin dye. The dye was stained onto the coated glass substrates depicted in Figure 11 which were then subjected to UV irradiation. If the

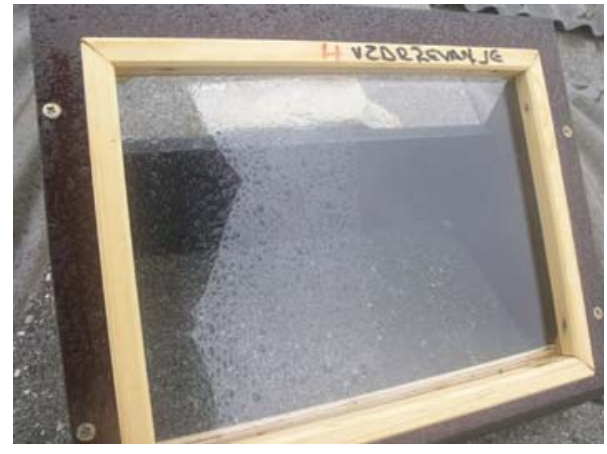

(a)

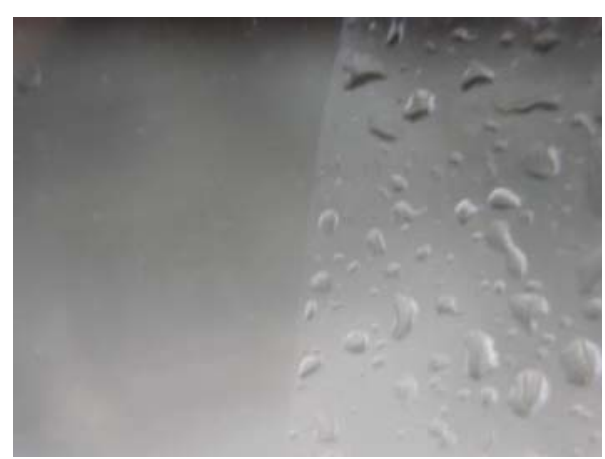

(b)

FIGURE 11: Glass substrates treated with self-cleaning coating and placed in real-life conditions. One can see that the coatings exhibit the superhydrophilic effect, which is depicted clearly in image (b), where a clear distinction between the treated (superhydrophilic) and untreated (water droplets) surface is shown.

$\mathrm{TiO}_{2}$ coating was to lose its activity or was even mechanically washed away due to improper and inadequate adhesion, the resazurin dye would not decompose and no observable change in colour would occur (Figure 12).

As seen from Figure 12, the resazurin test has proven that the $\mathrm{TiO}_{2}$ coating is still present and photocatalytically active after eight months of exposure to real-life weather conditions.

Nevertheless, it has to be noted that the contact-angle measurement technique that was used does not provide an entirely accurate impression on the photocatalytic activity of the coatings since the change in contact angle can also arise from the fact that $\mathrm{TiO}_{2}$ becomes superhydrophilic when illuminated with UV light. Therefore the change in contact angle value cannot be attributed to the methyl stearate decomposition solely, but also to the fact that the layer itself changes its surface properties. A more straightforward technique to determine the photocatalytic activity of the coatings should be used as will be the case in our future research activities of self-cleaning coatings.

\section{Conclusions}

In summary, anatase nanoparticles were synthesized based on a well-established sulphate process for $\mathrm{TiO}_{2}$ pigment production in which metatitanic acid is produced. The 


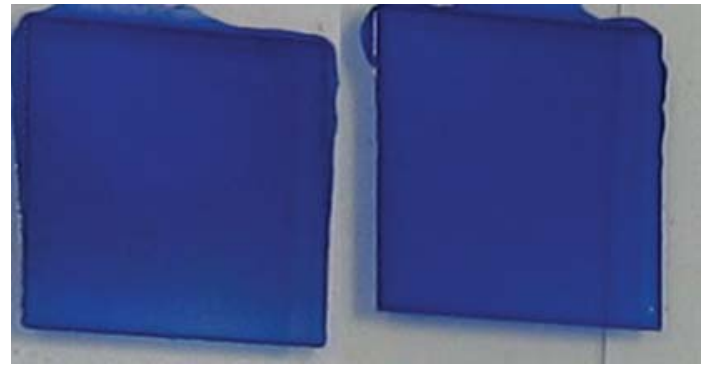

(a)

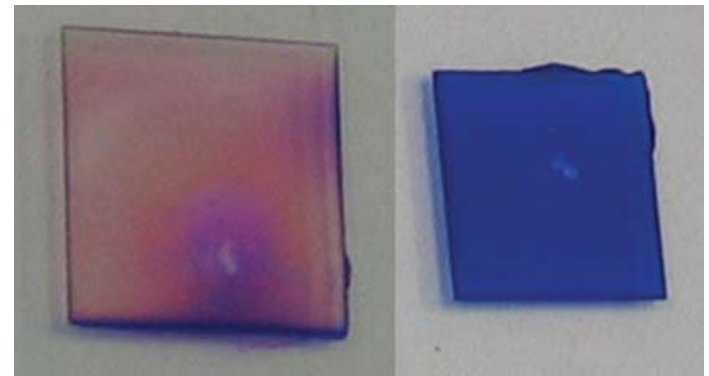

(c)

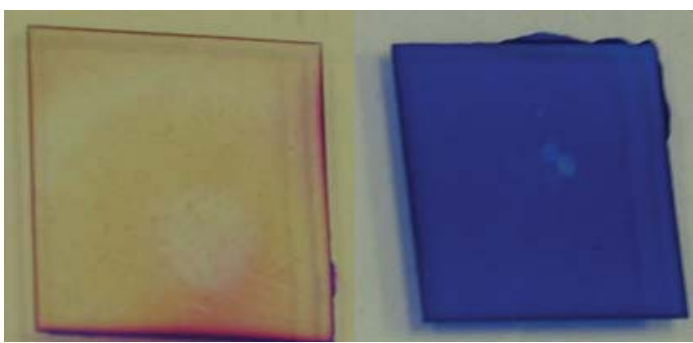

(e)

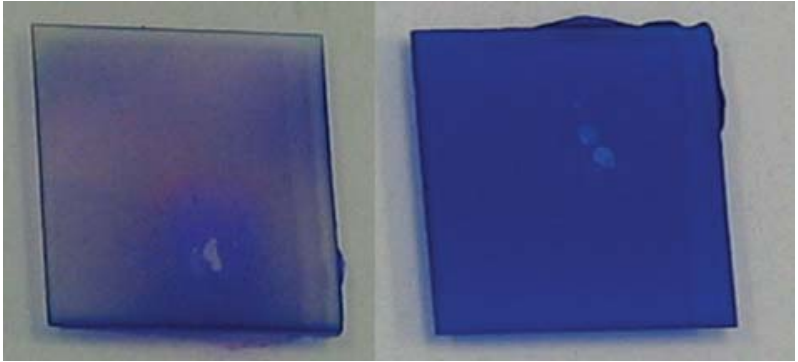

(b)

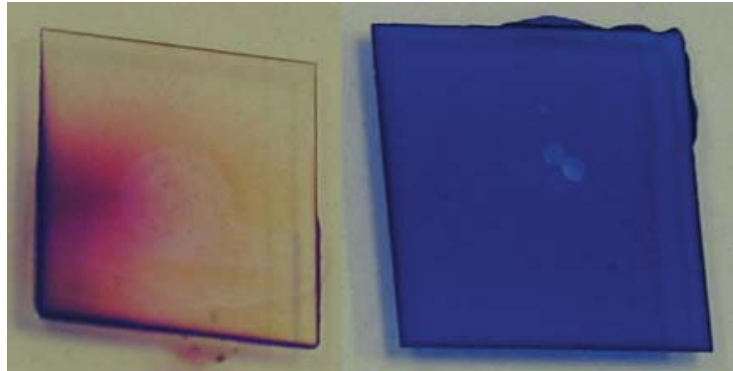

(d)

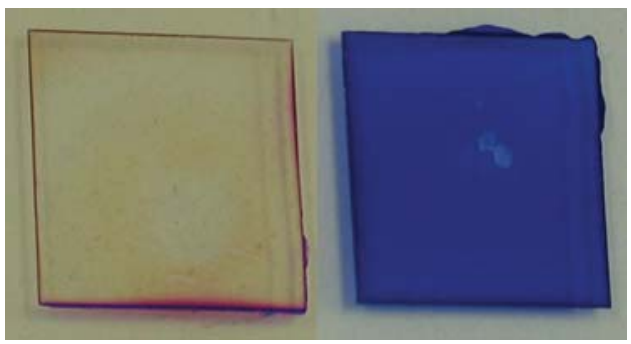

(f)

Figure 12: The resazurin decomposition test after (a) $0 \mathrm{~min}$, (b) $2.5 \mathrm{~min}$, (c) $5 \mathrm{~min}$, (d) $7.5 \mathrm{~min}$, (e) $12.5 \mathrm{~min}$ and (f) $20 \mathrm{~min}$. As can be seen, the resazurin dye decomposes when UV illumination is applied which clearly indicates that the $\mathrm{TiO}_{2}$ layer remained active and mechanically stable.

metatitanic acid can easily be converted into an anatase nanoparticulate suspension by a simple precipitation reaction using a water-soluble barium salt. The anatase nanoparticle suspension was used to prepare a self-cleaning-coating colloidal solution using a soft chemical method, which allows the production of transparent and highly photocatalytically active coatings. The coatings were tested using contactangle measurements and perform well even without thermal treatment, which enables a low-cost application on various substrates. The testing of self-cleaning coating is still in progress but shows great promise, since it exhibits good photocatalytic activity and mechanical characteristics. Furthermore, it is based on a wet/soft chemical method, which makes it suitable for large-scale production using inexpensive equipment and technology.

\section{Acknowledgments}

This work is partially financed by the European Union, European Social Fund and is based in part on the Ph.D. thesis of D. Verhovšek under Grant P-MR-07/26 by the TIA of Republic of Slovenia. The authors would also like to greatly acknowledge Cinkarna Celje Inc. which provided the raw material needed for performing the research presented in this paper. The electron microscopy was performed at the Center for Electron Microscopy at the Jožef Stefan Institute.

\section{References}

[1] J. Winkler, Titanium Dioxide, Vincentz, Hanover, Germany, 2003.

[2] B. O’Regan and M. Grätzel, "A low-cost, high-efficiency solar cell based on dye-sensitized colloidal $\mathrm{TiO}_{2}$ films," Nature, vol. 353, no. 6346, pp. 737-740, 1991.

[3] A. Jaroenworaluck, W. Sunsaneeyametha, N. Kosachan, and R. Stevens, "Characteristics of silica-coated $\mathrm{TiO}_{2}$ and its UV absorption for sunscreen cosmetic applications," Surface and Interface Analysis, vol. 38, no. 4, pp. 473-477, 2006.

[4] A. Fujishima and K. Honda, "Electrochemical photolysis of water at a semiconductor electrode," Nature, vol. 238, no. 5358, pp. 37-38, 1972. 
[5] S. U. M. Khan, M. Al-Shahry, and W. B. Ingler, "Efficient photochemical water splitting by a chemically modified n$\mathrm{TiO}_{2}$," Science, vol. 297, no. 5590, pp. 2243-2245, 2002.

[6] M. Schiavello, Photocatalysis and Environment: Trends and Applications, Kluwer Academic, Dodrecht, The Netherlands, 1988.

[7] N. Serpone and E. Pelizetti, Photocatalysis: Fundamentals and Applications, Wiley, New York, NY, USA, 1989.

[8] D. F. Ollis and H. Al-Ekabi, Photocatalytic Purification and Treatment of Water and Air, Elsevier, Amsterdam, The Netherlands, 1993.

[9] M. R. Hoffmann, S. T. Martin, W. Choi, and D. W. Bahnemann, "Environmental applications of semiconductor photocatalysis," Chemical Reviews, vol. 95, no. 1, pp. 69-96, 1995.

[10] J. M. Herrmann, "Heterogeneous photocatalysis: fundamentals and applications to the removal of various types of aqueous pollutants," Catalysis Today, vol. 53, no. 1, pp. 115129, 1999.

[11] D. Bahnemann, "Photocatalytic detoxification of polluted waters," in Environmental Photochemistry, Springer, Berlin, Germany, 1999.

[12] A. Fujishima, K. Hashimoto, and T. Watanabe, $\mathrm{TiO}_{2}$ Photocatalysis: Fundamentals and Applications, BKC, Tokyo, Japan, 1999.

[13] P. Pichat, J. Disdier, C. Hoang-Van, D. Mas, G. Goutailler, and C. Gaysse, "Purification/deodorization of indoor air and gaseous effluents by $\mathrm{TiO}_{2}$ photocatalysis," Catalysis Today, vol. 63, no. 2-4, pp. 363-369, 2000.

[14] M. Muneer, S. Das, V. B. Manilal, and A. Haridas, "Photocatalytic degradation of waste-water pollutants: titanium dioxide-mediated oxidation of methyl vinyl ketone," Journal of Photochemistry and Photobiology A, vol. 63, no. 1, pp. 107-114, 1992.

[15] S. G. Botta, D. J. Rodríguez, A. G. Leyva, and M. I. Litter, "Features of the transformation of HgII by heterogeneous photocatalysis over $\mathrm{TiO}_{2}$," Catalysis Today, vol. 76, no. 2-4, pp. 247-258, 2002.

[16] S. C. Moon, Y. Matsumura, M. Kitano, M. Matsuoka, and M. Anpo, "Hydrogen production using semiconducting oxide photocatalysts," Research on Chemical Intermediates, vol. 29, no. 3, pp. 233-256, 2003.

[17] J. H. Carey, J. Lawrence, and H. M. Tosine, "Photodechlorination of PCB's in the presence of titanium dioxide in aqueous suspensions," Bulletin of Environmental Contamination and Toxicology, vol. 16, no. 6, pp. 697-701, 1976.

[18] S. Sathyamoorthy, G. D. Moggridge, and M. J. Hounslow, "Controlling particle size during anatase precipitation," AIChE Journal, vol. 47, no. 9, pp. 2012-2024, 2001.

[19] S. Sathyamoorthy, G. D. Moggridge, and M. J. Hounslow, "Particle formation during anatase precipitation of seeded titanyl sulfate solution," Crystal Growth and Design, vol. 1, no. 2, pp. 123-129, 2001.

[20] U. Černigoj and U. L. Štangar, "Preparation of $\mathrm{TiO}_{2} / \mathrm{SiO}_{2}$ and use thereof for deposition of self-cleaning anti-fogging," International patent application number PCT/SI2009/000052. International patent publication number WO 2010/053459 A1; 2009.

[21] U. Černigoj, M. Kete, and U. L. Štangar, "Development of a fluorescence-based method for evaluation of self-cleaning properties of photocatalytic layers," Catalysis Today, vol. 151, no. $1-2$, pp. 46-52, 2010.

[22] U. Černigoj, M. Kete, U. L. Štangar, and V. Ducman, "Testing of photocatalytic activity of self-cleaning surfaces," Advances in Science and Technology, vol. 68, pp. 126-134, 2010.
[23] Y. Li, Y. Fan, and Y. Chen, "A novel method for preparation of nanocrystalline rutile $\mathrm{TiO}_{2}$ powders by liquid hydrolysis of $\mathrm{TiCl}_{4}$," Journal of Materials Chemistry, vol. 12, no. 5, pp. 13871390, 2002.

[24] J. W. Mullin, Cystallization, Butterworth-Heinemann, London, UK, 3rd edition, 1993.

[25] G. D. Parfitt, "Surface chemistry of oxides," Pure and Applied Chemistry, vol. 48, pp. 415-418, 1976.

[26] G. D. Parfitt, J. Ramsbotham, and C. H. Rochester, "An electrophoretic investigation of the effect of chloride and of silanol groups on the properties of the surface of rutile," Journal of Colloid And Interface Science, vol. 41, no. 3, pp. 437444, 1972.

[27] T. W. Evans, A. F. Sarofim, and G. Margolis, "Models of secondary nucleation attributable to crystal-crystallizer and crystal-crystal collisions," AIChE Journal, vol. 20, no. 5, pp. 959-966, 1974.

[28] E. G. Denk and G. D. Botsaris, "Mechanism of contact nucleation," Journal of Crystal Growth, vol. 15, no. 1, pp. 5760, 1972.

[29] R. Y. Qian and G. D. Botsaris, "A new mechanism for nuclei formation in suspension crystallizers: the role of interparticle forces," Chemical Engineering Science, vol. 52, no. 20, pp. 34293440, 1997. 


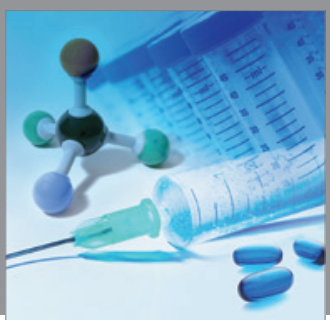

International Journal of

Medicinal Chemistry

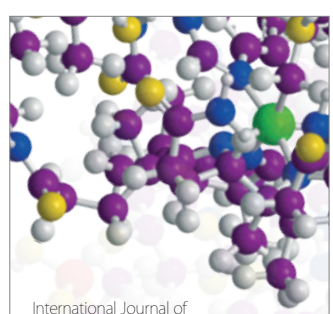

Carbohydrate Chemistry

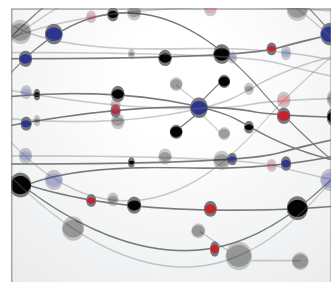

The Scientific World Journal
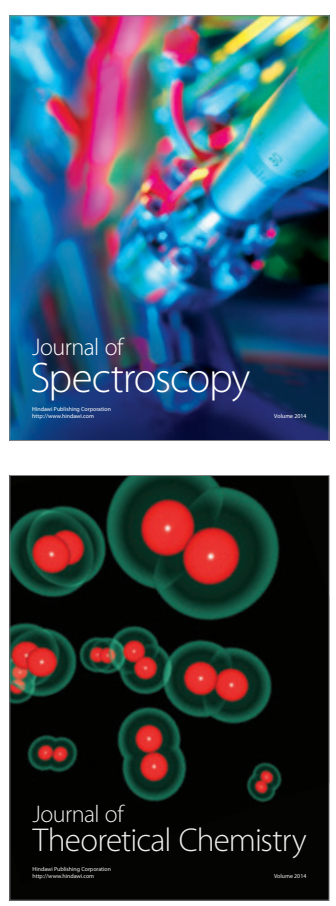
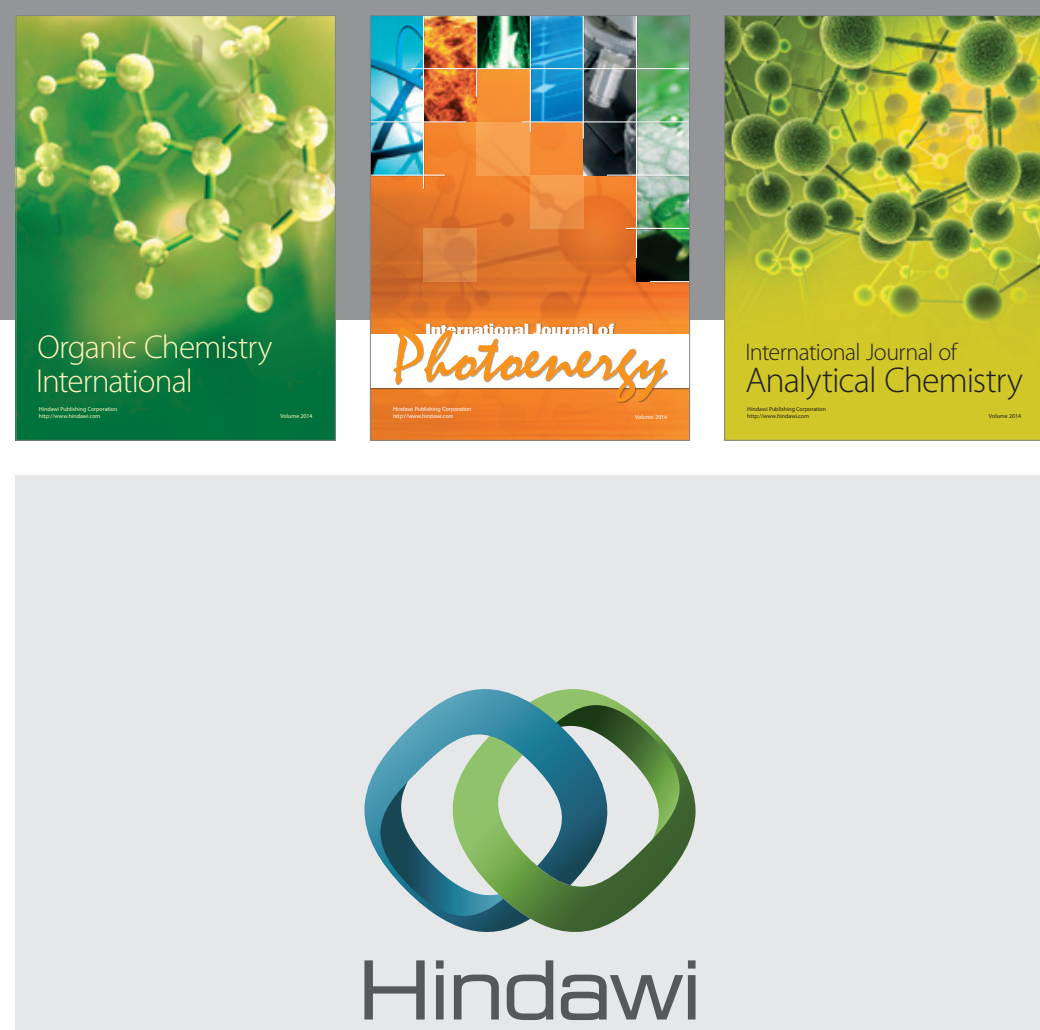

Submit your manuscripts at

http://www.hindawi.com
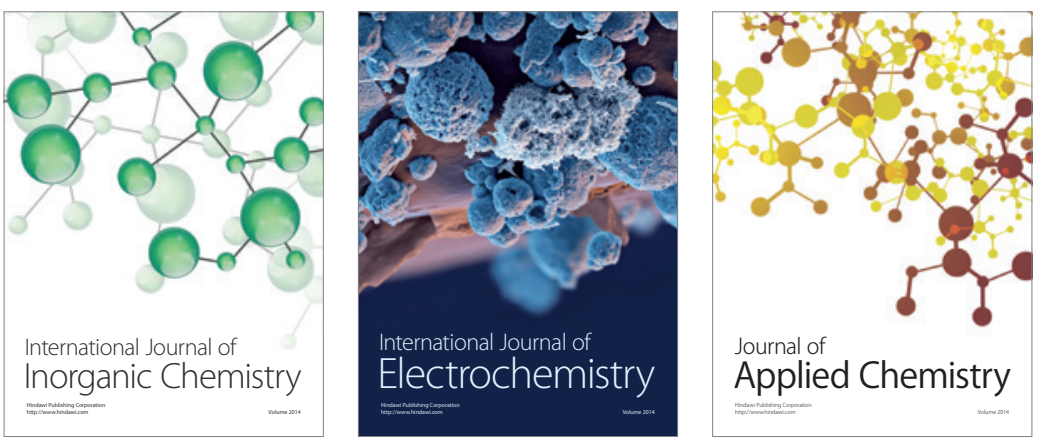

Journal of

Applied Chemistry
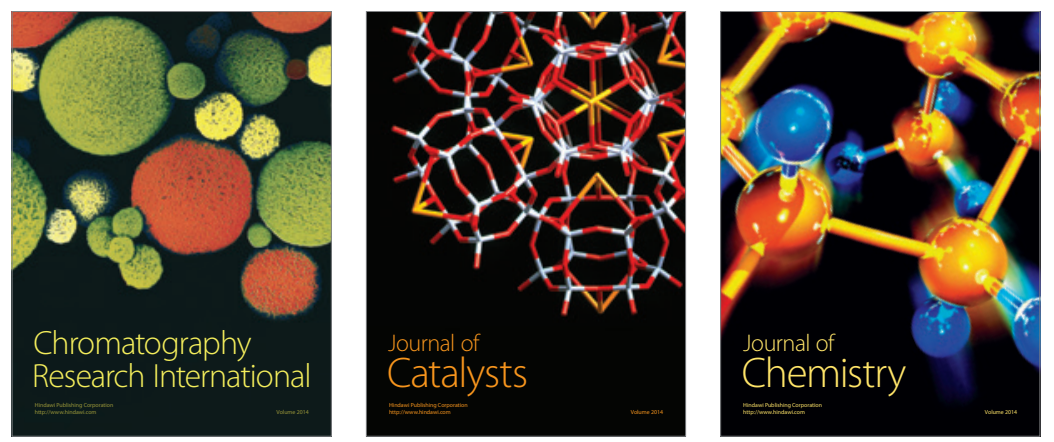
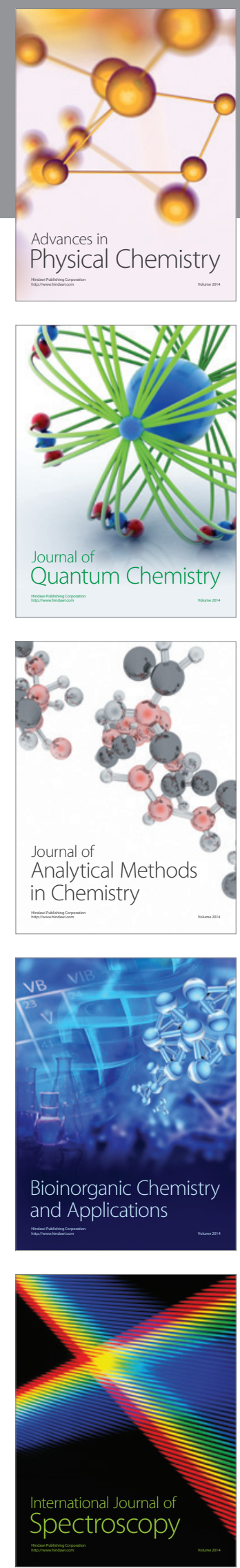